\title{
How do constituent quarks arise in QCD? Perturbation theory and the infra-red*
}

\author{
E. Bagan ${ }^{\mathrm{a}}$, M. Lavelle ${ }^{\mathrm{a}}$, D. McMullan ${ }^{\mathrm{b}}$, B. Fiol ${ }^{\mathrm{a}}$ and N. Roy ${ }^{\mathrm{a}}$ \\ ${ }^{a}$ Grup de Física Teòrica, Departament de Física and IFAE, \\ Edifici Cn, Universitat Autònoma de Barcelona \\ E-08193 Bellaterra (Barcelona) Spain \\ ${ }^{\mathrm{b}}$ Department of Mathematics and Statistics, \\ University of Plymouth, Drake Circus, \\ Plymouth, Devon PL4 8AA, United Kingdom
}

\begin{abstract}
We motivate the use of dressed charges by arguing that such objects are needed to describe, e.g., constituent quarks and, in general, physical charged states in gauge theories. We give a short introduction to dressings in both QED and QCD. We put special emphasis on the infra-red properties of a moving dressed charge. To be more precise, we demonstrate that the one loop propagator of a relativistic dressed charge can be renormalized in the mass shell scheme with no infra-red divergence showing up.
\end{abstract}

\section{WHY DRESSINGS?}

Many aspects of hadronic physics can be well described in terms of constituent quarks. The role played by such objects in our discovery of colour and QCD is well known. However, we do not yet have a good understanding of how such objects can emerge from QCD. This talk describes a new approach to this problem (for a review see [1]).

Any description of a (colour) charged particle in a field theory has to fulfill certain requirements: $(i)$ we need to include a chromo-(electro) magnetic cloud around the charge. This is known to underlie the infra-red problem and we recall the long postulated link between the infrared structure of QCD and confinement; (ii) since constituent quarks have, in the realm where the quark model is valid, a physical meaning, it is essential to describe them in a gauge invariant way. This, in turn, ensures that the constituent quarks have a well defined colour charge as required by the standard quark model[1].

\subsection{QED}

In QED, an approach which incorporates the above requirements was originally proposed by

\footnotetext{
*Talk presented by E. Bagan
}

Dirac|22. If some function $f_{\mu}(z, x)$ satisfies $\partial_{\mu}^{z} f^{\mu}(z, x)=\delta^{(4)}(z-x)$, then a charged particle at $x$ with an electromagnetic cloud around it may be written in a gauge invariant manner as

$\psi_{\mathrm{f}}(x)=\exp \left\{i e \int d^{4} z f_{\mu}(z, x) A^{\mu}(z)\right\} \psi(x)$.

The phase factor in (11) is usually called the dressing. There are as many dressings as there are possible choices of $f_{\mu}$ in (1). We will study two such choices. Our first example (which we will refer to as (i) below) is

$$
\begin{aligned}
& f_{0}=f_{1}=f_{2}=0 \\
& f_{3}(z, x)=-\theta\left(x^{3}-z^{3}\right) \prod_{j=0}^{2} \delta\left(z^{j}-x^{j}\right)
\end{aligned}
$$

which can be easily seen to correspond to a string attached to the charge at $x$ and going along a straight line in the $x^{3}$ direction out to infinity. One could equally well choose a more complicated path $\Gamma$ and in general we call such a dressed field, $\psi_{\Gamma}$. Example $(i i)$, which is the case we are primarily concerned with, is

$$
\begin{aligned}
& f_{0}=0, \\
& \vec{f}=\frac{1}{4 \pi} \delta\left(z^{0}-x^{0}\right) \vec{\nabla}_{z} \frac{1}{|\vec{z}-\vec{x}|},
\end{aligned}
$$


from which we find the dressed field

$\psi_{c}(x)=\exp \left\{i e \frac{\partial_{i} A_{i}}{\nabla^{2}}(x)\right\} \psi(x)$.

Example $(i)$ is not well suited to describe physical charges. First of all it corresponds to a very singular field configuration. Second, its path dependence is difficult to interpret and, finally, it has been shown that this field configuration is unstable [3]. Essentially what happens is that the charge generates a Coulombic field and the string radiates away to infinity. Fig. 11 shows the time evolution of a similar situation: the electric field is initially concentrated in the straight line joining two charges in such a way that Gauß's law holds. Again the string radiates away and only the dipole field generated by the charges remains in the far future 4 .

In contrast, example (ii) has very nice properties: $(a)$ it is stable; $(b)$ it is possible to factor out the path dependence in $\psi_{\Gamma}$ giving $\psi_{c}$, i.e., $\psi_{\Gamma}=N_{\Gamma} \psi_{c}$ where $N_{\Gamma}$ is a gauge invariant, but path dependent, factor; $(c)$ using the fundamental commutation relations one has

$\left[\vec{E}(\vec{x}), \psi_{c}(\vec{y})\right]=\frac{e}{4 \pi} \frac{\vec{x}-\vec{y}}{|\vec{x}-\vec{y}|^{3}} \psi_{c}(\vec{y})$,

where we recognize the factor before $\psi_{c}$ as the electric Coulomb field. This immediately suggests that example (ii) is the right dressing for a static charge.

One can generalize this dressing to the case of a charge moving with arbitrary velocity $\vec{v}$

$\psi_{v}=\exp \left\{i e \frac{g^{\mu \nu}-(\eta+v)^{\mu}(\eta-v)^{\nu}}{\partial^{2}-(\eta \cdot \partial)^{2}+(v \cdot \partial)^{2}} \partial_{\nu} A_{\mu}\right\} \psi$,

where $v=(0, \vec{v})$, and $\eta=(1, \overrightarrow{0}) ;(d)$ One can perform perturbative calculations using these dressings. We shall do this in the next section where we also show that the (one loop) mass shell renormalized dressed electron propagator,

$i S_{v}(p)=\int d^{4} x \exp \{i p \cdot x\}\left\langle 0\left|\psi_{v}(x) \bar{\psi}_{v}(0)\right| 0\right\rangle$,

is infra-red finite provided $p=m \gamma(1, \vec{v})[5]$, 6 , as was already predicted in Ref.[1]. As far as we know, there are only two other gauges with an infra-red finite charge propagator in the mass

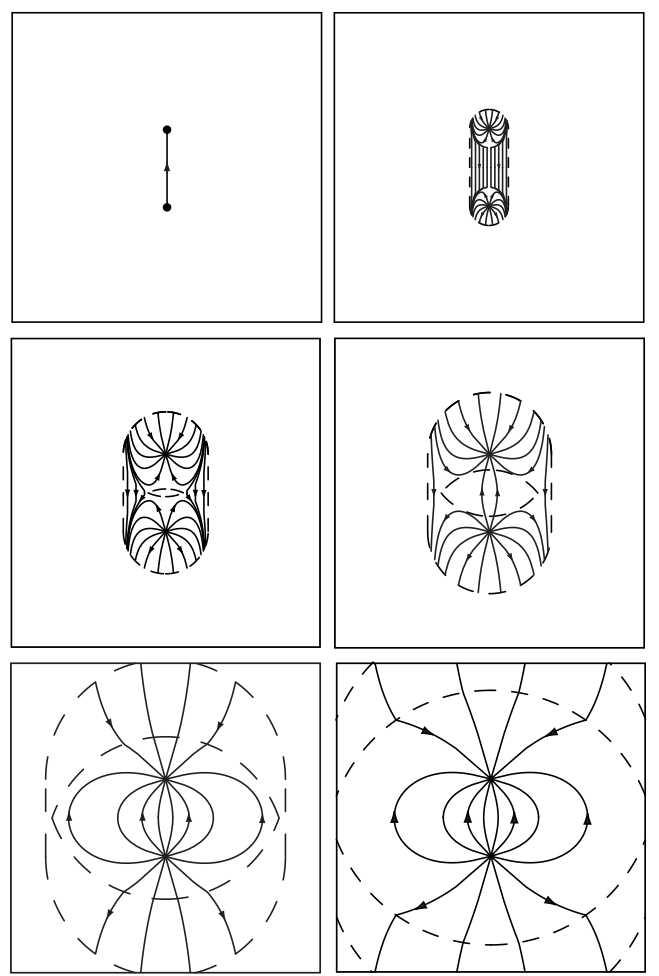

Figure 1. Six frames 4 of the time evolution of a electric field initially located in the straight line joining two opposite (static) charges. The dashed lines show the extension of the region where the radiation field from the decay of the string is present. Only the dipole field survives if we wait long enough.

shell scheme: the Yennie gauge and the Coulomb gauge. The latter is a particular case of our approach $(\vec{v} \rightarrow \overrightarrow{0})$. The infra-red finiteness of $S_{v}(p)$ can be understood as a consequence of having the charge dressed with the asymptotic electromagnetic field of a classical charge moving with velocity $\vec{v}$. This is a boosted Coulomb field. Since the infra-red behaviour is related to its slow fall-off, one would expect that the same dressing should lead to infra-red finite results also for scalar QED. This has been verified explicitly. The lack of infra-red divergences in the propaga- 
tor of the dressed charge is a necessary and highly non-trivial requirement for the construction of an asymptotic state with sharp momentum that can be interpreted as a (single) physical charge.

Before closing this section, we would like to comment on some subtleties associated with charged states. Note that $\psi_{v}$ is both non local and non covariant, which one might regard as a problem. However, it can be proved that these are unavoidable features of any operator that creates charged physical states out of the vacuum [1, , 7, 8]. Note also that Eq.(6) is not just a Lorentz boost of Eq.(14). This is a consequence of the lack of covariance and locality necessarily associated with charged states 1].

\subsection{QCD}

All the properties that have been discussed in the previous section go through to QCD in perturbation theory. It is also possible to define dressed gluon fields perturbatively. A new reason for introducing dressings in non-abelian gauge theories is that the colour charge is only welldefined on gauge invariant states such as a dressed quark[i]. However, it has been shown that beyond perturbation theory the Gribov ambiguity obstructs the construction of dressings|11. As a result, one cannot obtain any true observable out of a single lagrangian (quark) field. Therefore, our approach explains confinement in the sense that one cannot construct an asymptotic quark field.

\section{THE DRESSED ELECTRON/QUARK PROPAGATOR}

The Feynman diagrams for the one loop dressed propagator, $S_{v}(p)$, are shown in Fig.2. The diagram of Fig.2. a gives the standard self energy. The other three diagrams, Figs.2. b - d, contain a new vertex (the black box). The corresponding Feynman rule, which can be easily obtained from the perturbative expansion of Eq.(6), reads

$e \frac{(\eta+v)_{\mu}(\eta-v)_{\rho}-g_{\mu \rho}}{k^{2}-(k \cdot \eta)^{2}+(k \cdot v)^{2}} k^{\rho}$,

where $k(\mu)$ is the momentum (Lorentz index) of the incoming photon. We can now proceed in

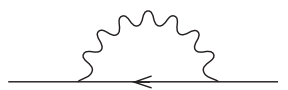

a

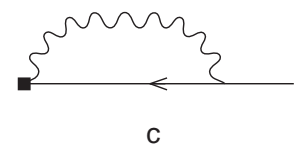

C

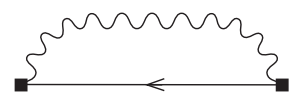

b

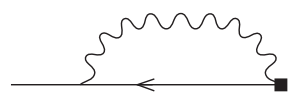

d
Figure 2. The diagrams which yield the one loop dressed propagator, $S_{v}(p)$. The vertices coming from the perturbative expansion of the dressing are denoted by a black box

two ways: (i) compute the diagrams of Figs.2. a$\mathrm{d}$ in a Feynman gauge and check that the result (before integrating the loop momentum) is independent of the gauge parameter; (ii) use the so called dressing gauge, in which the dressing phase is 1 , i.e., $\psi_{v}=\psi$ and compute only the diagram of Fig.2. a. In the dressing gauge the photon propagator is

$$
\begin{aligned}
& \frac{1}{k^{2}}\left\{-g_{\mu \nu}+\frac{k^{2}-[k \cdot(\eta-v)]^{2} \gamma^{-2}}{\left[k^{2}-(k \cdot \eta)^{2}+(k \cdot v)^{2}\right]^{2}} k_{\mu} k_{\nu}\right. \\
& \left.-\frac{k \cdot(\eta-v)}{k^{2}-(k \cdot \eta)^{2}+(k \cdot v)^{2}} k_{(\mu}(\eta+v)_{\nu)}\right\} \cdot(9
\end{aligned}
$$

Note that in the limit $\vec{v} \rightarrow \overrightarrow{0}$ this reduces to the propagator in Coulomb gauge. We have explicitly checked that the two procedures give the same loop momentum integral.

To integrate the loop momentum we have chosen to work in dimensional regularization with a space-time dimension $D=4-2 \epsilon$. This regularizes both the ultra-violet and infra-red divergences. In particular, the latter show up as $\int_{0}^{1} d u u^{D-5} \sim 1 / \epsilon$, where $u$ is a Feynman parameter. 


\subsection{Ultra-violet divergences}

The ultra-violet divergent part of the electron self-energy has the following structure

$$
\begin{aligned}
\Sigma_{U V} \sim & \frac{1}{\epsilon}\left\{-3 m+(\not p-m) \mathcal{F}_{1}(\vec{v})\right. \\
+ & \left.2[p \cdot v \not h-p \cdot \eta \psi] \mathcal{F}_{2}(\vec{v})\right\},
\end{aligned}
$$

where $\mathcal{F}_{1}$ and $\mathcal{F}_{2}$ do not depend on the external momentum, $p$. The last term in Eq.(10) seems to endanger the multiplicative renormalization of the propagator. However, one can check that Eq. (10) can be cancelled by introducing the standard mass shift, $m \rightarrow m-\delta m$, and the following multiplicative matrix renormalization for the electron

$\psi_{v}^{(\text {bare })}=\sqrt{Z_{2}} \exp \left\{-i \frac{Z^{\prime}}{Z_{2}} \sigma^{\mu \nu} \eta_{\mu} v_{\nu}\right\} \psi_{v}$,

which is reminiscent of a naive Lorentz boost upon a fermion.

\subsection{Renormalization}

To actually compute $\delta m, Z^{\prime}$ and $Z_{2}=1+\delta Z_{2}$ it is convenient to write the renormalized self energy as

$-i \Sigma=m \alpha+\not p \beta+p \cdot \eta \not h \delta+m \psi \varepsilon$,

where $\alpha, \beta, \delta$ and $\varepsilon$ depend upon $p^{2}, p \cdot \eta, p \cdot v$ and $v$ and they also contain the counterterms $\delta m, Z^{\prime}$ and $\delta Z_{2}$.

We recall that in the mass shell scheme one has to impose the following two conditions: $(i)$ The propagator has a simple pole at $m$, i.e., $m$ is the physical mass of the fermion; (ii) the residue of the propagator at $m$ is unity. From these two conditions it must be possible to determine $\delta m$, $Z^{\prime}$ and $\delta Z_{2}$. Condition ( $i$ i) implies that the renormalized $\Sigma$ must obey

$\tilde{\alpha}+\tilde{\beta}+\frac{(p \cdot \eta)^{2}}{m^{2}} \tilde{\delta}+\frac{p \cdot v}{m} \tilde{\varepsilon}=0$,

where the tildes signify that we put the momentum $p^{2}$ on shell: $p^{2}=m^{2}$. Note that $Z^{\prime}$ and $\delta Z_{2}$ do not enter in (13) so just $\delta m$ is determined. We find

$\delta m=\frac{e^{2}}{(4 \pi)^{2}}\left(\frac{3}{\epsilon}+4\right)$.
It is important to emphasize that this is the standard result for the mass shift and that it solves Eq.(13) for arbitrary $p \cdot \eta, p \cdot v$ and $v$.

Condition ( $i$ ) can only be satisfied if $p=$ $m \gamma(\eta+v)=m \gamma(1, \vec{v})$, which is precisely the momentum of the real electron moving with velocity $\vec{v}$. In addition we need that

$0=2 m^{2} \bar{\Delta}+\bar{\beta}-\bar{\delta}$

$0=\gamma\left(2 m^{2} \bar{\Delta}+\bar{\beta}\right)-\bar{\varepsilon}$

$0=2 m^{2} \bar{\Delta}+\bar{\alpha}+2 \bar{\beta}$,

where

$\Delta=\frac{\partial \alpha}{\partial p^{2}}+\frac{\partial \beta}{\partial p^{2}}+\frac{(p \cdot \eta)^{2}}{m^{2}} \frac{\partial \delta}{\partial p^{2}}+\frac{p \cdot v}{m} \frac{\partial \varepsilon}{\partial p^{2}}$

and the bar upon functions denotes that they must be computed at $p=m \gamma(1, \vec{v})$. If we now explicitly separate out the contributions of $Z^{\prime}$ and $\delta Z_{2}$ from the rest (to which we give a subscript $\mathrm{R}$ ) then we find that (15) can be rewritten as

$$
\begin{aligned}
-i \delta Z_{2}+2 \vec{v}^{2} i Z^{\prime} & =2 m^{2} \bar{\Delta}+\bar{\beta}_{R}-\bar{\delta}_{R} \\
-i \delta Z_{2}+2 i Z^{\prime} & =\gamma\left(2 m^{2} \bar{\Delta}+\bar{\beta}_{R}\right)-\bar{\varepsilon}_{R} \\
-i \delta Z_{2} & =2 m^{2} \bar{\Delta}+\bar{\alpha}_{R}+2 \bar{\beta}_{R} .
\end{aligned}
$$

Since we have three equations and two unknowns $\left(Z^{\prime}\right.$ and $\delta Z_{2}$ ) one might worry that perhaps no solution exists. However, a unique solution exists for our choice of mass shell. It reads

$$
\begin{aligned}
Z^{\prime} & =\frac{1}{2 i}\left[\gamma^{2} \bar{\delta}_{R}-\gamma \bar{\epsilon}_{R}\right] \\
\delta Z_{2} & =-\frac{1}{i}\left[\bar{\alpha}_{R}+2 \bar{\beta}_{R}+2 m^{2} \bar{\Delta}\right] .
\end{aligned}
$$

As is the case for the standard fermion propagator, the infra-red singularities can only enter through derivatives with respect to $p^{2}$. Hence, $Z^{\prime}$ is infra-red finite and infra-red divergences can only arise in $\delta Z_{2}$ through $\bar{\Delta}$. The total infra-red divergent contribution to $\bar{\Delta}$ is

$$
\begin{aligned}
\bar{\Delta}_{\text {IR }} & \sim \int_{0}^{1} d u u^{D-5}\left\{-2+2 \int_{0}^{1} \frac{d x}{\sqrt{1-x} \sqrt{1-\vec{v}^{2} x}}\right. \\
& \times\left(1+\vec{v}^{2}-2 \vec{v}^{2} x\right) \\
& \left.-\gamma^{-2} \int_{0}^{1} \frac{d x x}{\sqrt{1-x} \sqrt{1-\vec{v}^{2} x}} \frac{3+\vec{v}^{2}-2 \vec{v}^{2} x}{2\left(1-\vec{v}^{2} x\right)}\right\} \\
& =0,
\end{aligned}
$$


and no infra-red divergence arises in the mass shell renormalize propagator of the dressed electron. The full expressions for $Z^{\prime}$ and $Z_{2}$ can be found in Ref.[6]. As we have already mentioned, one can also repeat the calculation in scalar QED where the algebra is not so heavy. Again one can renormalize the propagator of the dressed scalar electron, defined as in (6) replacing $\psi$ by the scalar field. In this case, no $Z^{\prime}$ is needed and we can get rid of the ultra-violet divergences through the usual counterterms $Z_{2}$ and $\delta m$. Again, no infra-red divergence arise. The consistency of these calculations with our expectations is compelling evidence for the validity of this approach.

\section{SUMMARY}

To conclude we note that

- Any description of a physical charge must be gauge invariant. Gauß's law implies an intimate link between charges and a chromo-(electro-) magnetic cloud.

- Not all gauge invariant descriptions are physically relevant. One needs to find the right ones.

- In QCD there is no such description of a single quark outside of perturbation theory. This sets the limits of the constituent quark model and fixes when jets start to hadronise.

- The perturbative tests reported here all worked. They are now being extended to vertex studies.

- Phenomenologically, the main question is: at what scale does the Gribov ambiguity prevent any description of a quark from being stable?

\section{Acknowledgments}

EB \& BF were supported by CICYT research project AEN95-0815 and ML by AEN95-0882. NR was supported by a grant from the region Rhône-Alpes.

\section{REFERENCES}

1. M. Lavelle and D. McMullan, Constituent Quarks from $Q C D$, to appear in Physics Reports C.

2. P.A.M. Dirac, Can. J. Phys. 33, 650 (1955)

3. L.V. Prokhorov, D.V. Fursaev and S.V. Shabanov, TMP 97, 1355 (1994)

4. A simulation of the evolution of such a state and a more detailed description of the underlying physics may be found at the web page http://www.ifae.es/ roy/

5. E. Bagan, M. Lavelle and D. McMullan, Phys. Lett. B 370, 128 (1996).

6. E. Bagan, M. Lavelle and D. McMullan, A Class of Physically Motivated Gauges with an Infra-Red Finite Electron Propagator, submitted for publication. (hep-th/9602083).

7. F. Strocchi and A. S. Wightman, J. Math. Phys. 15, 2198 (1974).

8. J. Fröhlich, G. Morchio and F. Strocchi, Ann. Phys. 119, 241 (1979). 